4. Подгорная А.С. Пролиферативные процессы эндометрия: современное состояние проблемы. Гомель: РНПЦ РМ и ЭЧ, 2018: 30.

5. Хмельницкий О. К. Цитологическая и гистологическая диагностика заболеваний шейки и тела матки. Санкт-Петербург: SOTIS, 2000. 333 c.

6. Predictive diagnosis of endometrial hyperplasia and personalized therapeutic strategy in women of fertile age / V. M. Goncharenko et al. URL: https:// link.springer.com/article/10.1186/1878- 5085-4-24

7. Beniuk V. O., Goncharenko V. M., Bubnov R. V., Melnychuk O. Updating personalized management algorithm of endometrial hyperplasia in premenopause women. EPMA Journal. 2016. Vol. 7, Suppl 1. A28. URL: http:// link.springer.com/article/10.1186/ s13167-016-0054-6. (16.09.2015)

8. Goncharenko V. M., Bubnov R. V. Endometrial hyperplasia in women of fertile age: personalized diagnosis and therapeutic strategy. 16th World Congress of the International Society of Gynecological Endocrinology (ISGE), Florence, Italy, March 2014. URL: http://www.epmajournal.com/content/4/ 1/24/ - ins1.

DOI https://doi.org/10.30525/978-9934-588-81-5-1.8

\title{
HISTOLOGICAL FEATURES OF CHRONIC GASTROINTESTINAL PATHOLOGY DEPENDING ON THE FORM OF THE DISEASE
}

\author{
Bobrova V. I. \\ $M D, P h D$, \\ Professor at the Pediatric Department № 1 \\ Bogomolets National Medical University \\ Proshchenko Y. I. \\ $P h D$, \\ Assistant at the Pediatric Department № 1 \\ Bogomolets National Medical University \\ Kyiv, Ukraine
}

The growth trend of children's gastroenterological pathology is an important medical and social problem and is associated with burdened heredity, stress, H.pylori infection, unbalanced nutrition, ulcerogenic effect of drugs, harmful habits (including tobacco smoking) and, as a consequence, disorders of secretory, motor and cytoprotective functions of the stomach. 32 
Chronic gastroduodenitis (CGD) is the most common form of chronic gastroduodenal disease in childhood, accounting 58-74\% of gastric and duodenum diseases. CGD - a chronic recurrent inflammatory disease, which is accompanied by non-specific structural adjustment of mucosa membrane (MM) and gastric and duodenal glandular apparatus (dystrophic, inflammatory and dysregenerating changes), disorder of secretory and motor function of the stomach and duodenal gland [1,2].

The aim of our work was to determine morphological features of the different form of chronic gastroduodenal pathology.

Materials and methods. There were 136 children aged from 12 to 17 years with verified CGD exacerbation hospitalized to the in-patient pediatric department of Kyiv city children’s hospital № 9. To verify the diagnosis all children underwent esophagogastroduodenoscopy (EGDS) and intragastric $\mathrm{pH}$-metry during first 3 days after hospitalization. To detect $H$. pylori contamination serological and histological methods were used. Coloration of tissues' slices with hematoxylin and eosin was used to evaluate histological changes of stomach mucosa (SM) and duodenum's mucosa (DM). The received results were interpreted according to "Sydney system". Statistic examination of the results was made with standard Microsoft Excel programs.

The results of the research and their discussion.

There were $82(60,3 \pm 4,2 \%)$ boys and $54(39,7 \pm 4,2 \%)$ girls among examined children. Among children with chronic gastroduodenal pathology, prevailed boys $(60.3 \pm 4.2 \%)$ aged $15-17$ years $(55.1 \pm 4.3 \%)$. The anamnesis study revealed that in most children the duration of the disease was more than 5 years $(52.9 \pm 4.3 \%)$. The most frequent exogenous factors that may have contributed to the recurrence of the disease in the children were: eating disorders $89.7 \pm 2.6 \%$; harmful habits, including smoking $33.8 \pm 4.1 \%$; food allergy $24.3 \pm 3.7 \%$. At the time of hospitalization, all children complained of abdominal pain (short-term acute pain of $46.3 \pm 4.3 \%$, non-intensive dull pain $53.7 \pm 4.3 \%$ ). In the course of comparative analysis it was found that the acute nature of pain syndrome was more frequently observed in patients with erythematous changes in MM, while the dull pain was more frequently observed in patients with destructive changes in SM and DM. Dyspeptic syndrome was observed in all patients. The leading symptoms in children were nausea $61(44.9 \pm 4.3 \%)$, hertburn $48(35.3 \pm 4.1 \%)$, reduced appetite $52(38.2 \pm 4.2 \%)$ and flatulence $57(41.9 \pm 4.2 \%)$. Among the manifestations of dyspeptic syndrome no reliable differences related to the form of the disease were found.

The result of endoscopic examination showed the predominance of erythematous changes of the MM of stomach and duodenum in the examined children. Hypertrophic gastropathy in combination with erythematous 
changes were observed in $16.2 \pm 3.2 \%$ of children. Erosion were found in $21.3 \pm 3.5 \%$ (including erosive gastritis combined with erythematous duodenitis in $3.7 \pm 1.6 \%$; erosive duodenitis combined with erythematous gastritis in $14 \pm 3.0 \%$ and erosive duodenitis combined with hypertrophic gastritis in $3.7 \pm 1.6 \%$ ). Ulcer of duodenum was diagnosed in $26.5 \pm 3.8 \%$ of children. In endoscopic examination in $55.1 \pm 4.3 \%$ of children were diagnosed disorder of motor function in the form of duodenogastral reflux (DGR) and gastroesophageal reflux (GER). Among the disorders of motor function, DGR was significantly more prevalent than GER $(36.0 \pm 4.1 \%$ and $19.1 \pm 3.4 \%$ ).

All the children were assessed for basal acidity. It was found that normal stomach acidity prevailed (47.06 $\pm 4.3 \%), 25(18.4 \pm 3.3 \%)$ children had hyperacid levels of basal secretion, and 47 (34.6 $\pm 4.1 \%)$ had hypoacidity. In a comparative analysis, it was found that erythematous changes in $80 \pm 17.9 \%$ and $57.1 \pm 13.2 \%$ prevailed in patients with elevated and normal gastric acidity, while hypertrophic gastropathy was diagnosed in children with reduced acidity $50 \pm 158 \%$. Destructive changes in the MM were significantly more frequently observed in patients with different disorders of acidity $(33.3 \pm 12.2 \%)$. Pathological changes in motor function were also more frequently observed in patients with impaired stomach acidity, for example DGR was diagnosed in $40 \pm 15.5 \%$ with hyperacidity and in $60 \pm 21.9 \%$ of patients with hypoacidity, while in patients with normal stomach acidity DGR was observed only in $21.4 \pm 11.0 \%$. GER was found only in patients with reduced and increased stomach acidity $(20 \pm 12.6 \%$ and $80 \pm 17.9 \%)$.

To verify CGDP histological investigation of antral stomach's and duodenum's mucosa was performed in all children. General evaluation of SM and DM morphologic changes on the first stage of investigation was made. According to the results, in $27(93,1 \pm 4,7 \%)$ children it was chronic nonatrophic gastritis detected, $24(82,8 \pm 4,0 \%)$ children - chronic non-atrophic duodenitis, $2(6,9 \pm 4,7 \%)$ patients - chronic atrophic gastritis and $5(17,2 \pm 7,0 \%)$ patients - chronic atrophic duodenitis found out. During the comparative analysis it was found out that certain patient with the background of endoscopic erythematous changes, not only inflammatory, but also erosive and atrophic processes of SM and DM were found out histologically.

The morphological study evaluated the inflammatory process depending on the presence and severity of lymph-plasmocytic, neutrophil infiltration of the own plate and fibrosis of MM: $37.9 \pm 9.0 \%$ had severe degree 
of inflammation, moderate - in $34.5 \pm 8.8 \%$ of patients and in $27.6 \pm 8.3 \%$ of patients - a mild degree of inflammatory changes in MM.

During the comparative analysis, it was found that severe degree of inflammation was observed in $50.0 \pm 20.4 \%$ of children with duodenal ulcer and in $100 \%$ of children with hypertrophic gastritis and erosive duodenitis. A moderate degree of inflammation activity was typical for hypertrophic gastritis and erythematous duodenitis $66.7 \pm 19.2 \%$, while a mild degree of inflammation was typical for patients with erythematous stomach changes and erosive duodenitis (picture 1). In $33.3 \pm 27.2 \%$ patient with hypertrophic gastropathy and erosive changes of the duodenal mucosa were diagnosed atrophy of the DM. (Fig. 2)

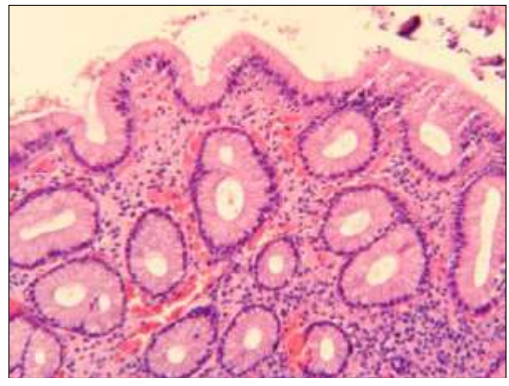

a)

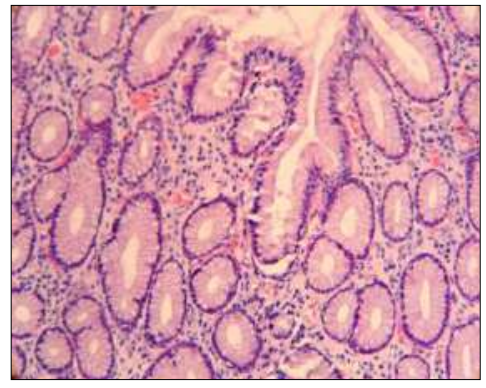

b)

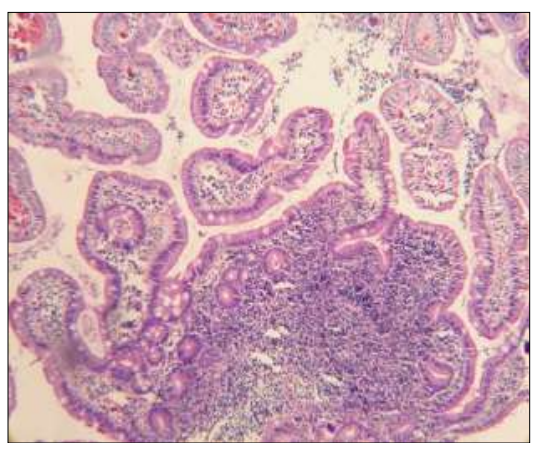

c)

Fig. 1. Microphoto of gastric mucosa's biopsy specimen: a - chronic nonatrophic gastritis, marked degree of inflammation; b - chronic nonatrophic gastritis, mild degree of inflammation; c - chronic duodenitis, hypotrophy of MM 
Fibrosis of stroma of MM was observed in $27.6 \pm 8.3 \%$ of patients. The comparative analysis revealed that fibrous changes were most common in children with ulcer disease of duodenum $(50.0 \pm 20.4 \%)$ and hypertrophic gastritis with erosive changes $(33.3 \pm 27.2 \%)$.

It should be noted that for majority of children the inflammatory process was accompanied by lymphocytic infiltration of MM, which testifies violation of physiological reparative processes and possible chronization of the inflammatory process, given that lymphocytes regulate the state of local immune protection and are able to accelerate fibroblastic processes and collagenesis. Neutrophilic and eosinophilic infiltrations of MM were more frequently noted in patients with hypertrophic changes in MM $(16.7 \pm 15.2 \%$ and $83.3 \pm 15.2 \%$ ), which indicates the activity of inflammatory process in MM. Microcirculatory changes in MM in the form of hemorrhages and microthrombosis were diagnosed among $88.9 \pm 10.5 \%$ of children with hypertrophic gastritis and in $92.9 \pm 6.9 \%$ of patients with ulcer disease of duodenum.

In order to diagnose the infection of children by H.pylori, the results of serological and histological methods were evaluated in our work. During the serological study it was found that among $51(37,5 \pm 4,2 \%)$ children the result was positive, weak positive - among $28(20,63,5 \%)$ children, negative - among $57(41,942 \%)$ children. Histologically, H.pylori infection was detected among $37.0 \%$ of children. During the comparative analysis it was found that H.pylori infection more often accompanies hypertrophic changes MM $77,8 \pm 13,9 \%$, besides it was noted that with this pathology among $85,7 \pm 13,2 \%$ of children were affected by $3 \mathrm{~d}$ degree of contamination with H.pylori bacteria.

Thus, the study showed that in case of destructive changes in MM, patients were significantly more concerned about the dull nature of the pain syndrome related to moderate and severe degree of inflammation of MM. Erythematous changes of MM are more often accompanied by the acute character of pain syndrome with mild degree of inflammation of MM, while hypertrophic changes of MM is characterized by moderate degree of inflammation with widespread fibrosis on the background of neutrophilic and eosinophilic infiltration of MM, expressed microcirculation disorders and $\mathrm{N}$. pylori contamination.

\section{References:}

1. Pentti Sipponen. Chronic gastritis / Pentti Sipponen, Heidi-Ingrid Maaroos. - Scand J Gastroenterol. 2015 Jun; 50(6):657-67.

2. Ya S Tsimmerman. Topical problems of chronic gastritis / Ya. S. Tsimmerman, Yu A Zakharova. Klin Med (Mosk). 2017; 95(1):8-14. 